\title{
Optimization and Characterization of Poly[R]hydroxyalkanoates of Pseudomonas aeruginosa
}

\author{
Antony V. Samrot, M. Bhakyalakshmi, K.L. Venkatraman, K. Sahiti, \\ Sheryl-AnnPhilip, T. Jahnavi and P. Senthilkumar
}

Department of Biotechnology, Sathyabama University, Sholinganallur, Chennai-600119, India.

DOI: http://dx.doi.org/10.13005/bbra/1883

(Received: 10 June 2015; accepted: 05 August 2015)

\begin{abstract}
Polynucleotides, polypeptides, polysaccharides are the polymers seen in living organisms. Many biopolymers are important reserve compounds that are stored in the cytoplasm as insoluble inclusions. Likewise Polyhydroxyalkanoates are natural, renewable biopolyester accumulated in bacteria in the presence of limited nitrogen sources and higher concentration of carbon source. In this study, PHA accumulation pattern of Pseudomonas aeruginosa was studied. The PHA was optimized for various carbon sources, incubation time, pH and temperature. Analytical techniques like FTIR and GC-MS were done to determine the types of monomer present in the biopolyester.
\end{abstract}

Key words: PHA - Polyhydroxyalkanoates, Pseudomonas aeruginosa, GC-MS, FTIR

Polyhydroxyalkanoate(PHA) are biopolyesters accumulated in microorganism when bacterial cells are grown in excess carbon sources and other nutrients at limited conditions. Polyhydroxy alkanoates (PHA) are biopolymers accumulated when bacterial cells are grown in excess carbon source and other nutrients at limited conditions as energy source at late stationary phase and reduces mortality rate ${ }^{1,2}$. PHAs are normally found as insoluble inclusions in cytoplasm thus known as PHA granules and these granules contain $97.5 \%$ PHA, $2 \%$ proteins and small amounts of lipids. PHA granule associated proteins plays an important role in PHA synthesis and granule formation ${ }^{3}$. The maximum yield of PHAs depends on several factors including stability and safety of the organism, growth and PHA accumulation rates, achievable cell densities

\footnotetext{
* To whom all correspondence should be addressed.
}

and PHAs content, extractability of the polymer, molecular weight of accumulated PHA, range of utilizable carbon sources, cost of carbon sources and other components of the medium and occurrence of the byproducts ${ }^{4}$. Major problem associated with the production of PHAs from bacteria are its cultural optimization studies. Optimization of the suitable carbon source in the medium is an important criteria for high production of PHA. By changing the carbon source and also the concentration of carbon source used in the fermentation process, it is possible to produce different PHAs with varied monomer content ranging from thermoplastic to rubbery polymers ${ }^{2}$. The physical parameters like incubation time, temperature and $\mathrm{pH}$ are also to be considered in PHA production studies. Microbial PHA production is highly influenced by $\mathrm{pH}$ of cultivation medium $^{5}$. In the earlier times the most common analytical technique used for PHA estimation was gravimetric method ${ }^{6}$. Several methods are now available for determination of the PHA contents of 
microorganisms, the composition of PHAs, and the distribution of the monomer units. The gas chromatographic (GC) identification of PHA components was later proposed by Braunegg et al., 7 . This method involves simultaneous extraction and methanolysis of PHA, in mild acid or alkaline conditions, to form hydroxyalkanoate methylesters which are then analysed by GC. This method is rapid (4 h), sensitive, reproducible and requires only small amount of samples. Other analytical techniques used for confirmation of PHA are FTIR, UV spectrometry.

There are two groups among the PHAs producing bacteria on the basis of number of carbon atoms present in monomer units ${ }^{8}$ i.e Shortchain length PHAs(3-5 carbon atoms) and medium chain length PHAs(6-4 carbon atoms) ${ }^{9}$. Most organisms synthesize either SCL-PHA primarily having 3HB units or MCL-PHA having 3-hydroxy octanoate. The co-polymers of PHA are also produced which varies according to substrates used. These co-polymers of SCL-PHA and MCLPHA has got more tensile strength and other mechanical properties. PHA can be synthesized either by chemical methods or by biological synthesis. The biological synthesis alone yields much higher molecular weight than chemical method as these PHA can also be produced by chemical method. The biosynthesis of different types of PHAs significantly depends on the chosen microorganisms, fermentation condition and different carbon sources. The vast majority of microbes synthesize either SCL-PHA containing primarily 3-hydroxyoctanoate (3HO) and 3hydroxydecanoate (3HD) as the major monomers ${ }^{2,9,10}$

\section{MATERIALS AND METHODS}

\section{Isolation of the bacterial strain}

The bacterial strain were isolated from the dairy waste and soil. $1 \mathrm{~g}$ of soil sample were serially diluted. After serial dilution, $1 \mathrm{ml}$ of the culture was introduced into sterile nutrient agar. Plates were incubated for 24 hours and observed for colony formation. The organisms grown were inoculated into cetrimide agar and incubated for 24 hours at $37^{\circ} \mathrm{C}$. Thus obtained pure culture was stored in nutrient agar slant containing 25\% glycerol solution at $-20^{\circ} \mathrm{C}$ for further use.

\section{Production and extraction of PHA}

The organism was inoculated into minimal media containing $\left(\mathrm{gl}^{-1}\right) \mathrm{K}_{2} \mathrm{HPO}_{4}-3 \mathrm{~g}$, $\mathrm{Na}_{2} \mathrm{HPO}_{4}-6 \mathrm{~g}, \mathrm{NH}_{4} \mathrm{Cl}-2 \mathrm{~g}, \mathrm{NaCl}-5 \mathrm{~g}, \mathrm{MgSO}_{4}-1 \mathrm{~g}$, $\mathrm{CaCl}_{2}-0.1 \mathrm{~g}$ and $\mathrm{FeCl}_{3}-0.06 \mathrm{~g}$ and it was incubated at $37^{\circ} \mathrm{C}$ for 48 hours under aerobic agitation of 150 rpm. The fermented culture was centrifuged at 10,000rpm for 25 minutes at $4{ }^{\circ} \mathrm{C}$. The cell pellet was washed twice with distilled water. Cell pellet was dried in Hot air oven. $3 \mathrm{ml}$ of $4 \%$ Sodium hypochlorite and $7 \mathrm{ml}$ of Chloroform was added to $1 \mathrm{~g}$ dried biomass and agitated vigorously. The mixture was then centrifuged at 2500rpm for 5 minutes to get three separate layers. The bottom layer contains chloroform with dissolved PHAs. PHA was obtained by adding methanol drop-wisedrop to get white precipitate. The precipitate was collected and added with $1 \mathrm{ml}$ Chloroform and transferred to an eppendorf tube, air-dried to get pure PHA. PHA \% was calculated using dry weight of PHA and Cell Dry Weight ${ }^{11}$.

\section{Optimization of PHA production}

The production of PHA was optimized with respect to five different carbon source such as glucose, fructose, maltose, sucrose and lactose at various concentrations ${ }^{12,13}$. The optimum conditions like incubation time, $\mathrm{pH}$ and temperature for PHA accumulation were also determined.

\section{Characterization of PHA \\ UV spectroscopy}

The PHA was added with $10 \mathrm{ml} \mathrm{H}_{2} \mathrm{SO}_{4}$ and heated at $100^{\circ} \mathrm{C}$ for $10 \mathrm{~min}$. Absorbance between 200 and $400 \mathrm{~nm}$ was recorded in UV spectroscopy, peak between 230 and $240 \mathrm{~nm}$ confirms the presence of $\mathrm{PHA}^{14}$.

\section{Thin layer chromatography}

The presence of PHA was confirmed by thin layer chromatography based on the method of Panda et al., ${ }^{15}$.

\section{FTIR}

The extracted PHA was dissolved in chloroform and Infrared spectra (IR) (4000 to 400 $\mathrm{cm}^{-1}$ ) were recorded on polymer films cast from chloroform solution onto $\mathrm{KBr}$ plates using FTIR (Shimadzu, DR-800) at $27^{\circ} \mathrm{C}^{16}$.

\section{GC-MS analysis}

GC analysis of powdered PHA was carried out by the method described by Brandl et al., ${ }^{17}$. The 2 methyl esters were analyzed by GC clarus 500 Perkin Elmer detector in a 30m x 0.25 mm ID x 
1ìm df capillary column was used. Helium (1 ml/ min) was used as carrier gas. The injector and detector are at $250^{\circ} \mathrm{C}$ and $200^{\circ} \mathrm{C}$ respectively.

\section{RESULT SAND DISCUSSION}

\section{Isolation and identification of organism}

The isolated organism was found to be Pseudomonas aeruginosa as it was able to produce pigment on cetrimide agar (Fig. 1). A similar study was done by Bhuwal et al., ${ }^{18}$ who isolated PHA producing organism from cardboard manufacturing industry waste water.

Pseudomonas aeruginosa accumulated more PHA in glucose containing medium (Fig.2). Nisha et al., ${ }^{16}$ also found Pseudomonas putida to accumulate more PHA in glucose containing medium only. Highest accumulation was observed at 48 hours in minimal media. The organism was found to accumulate higher percentage of PHA in glucose containing medium. PHA producing bacteria can be divided into two groups based on the culture media .In the first group ,the bacteria accumulates PHA at exponential phase only if there is a limited amount of nutrients (eg, Pseudomonas oleovorans) ${ }^{19}$.The second group, accumulates PHA during growth and it does not requires nutrient limitation(e.g. recombinant E.coli) ${ }^{20}$. All the organisms are tends to accumulate PHA at the stationary phase ${ }^{12}$. Even Jenifer et al., ${ }^{13}$ found Agrobacterium tumefaciens SU-11 to accumulate at $48 \mathrm{~h}$ of incubation. But this was contradictory to the result of Nisha et al., ${ }^{16}$ who found Pseudomonas putida SU-8 to accumulate in early log phase.

Pseudomonas aeruginosa was found to accumulate higher concentration of PHA at pH 8. Samrot et al. ${ }^{12}$ and Nisha et al. ${ }^{16}$ found Enterobacter cloacae SU-1 and Pseudomonas putida SU-8 to accumulate PHA near pH7 (Fig.4). Pseudomonas aeruginosa was found to accumulate higher concentration of PHA at $40^{\circ} \mathrm{C}$ (Fig.5). Optimal condition for PHA accumulation by Pseudomonas aeruginosa were at glucose containing medium, $\mathrm{pH}$, temperature at $40^{\circ} \mathrm{C}$. A similar study on optimization of PHA was done by Prasad. and Sethi ${ }^{21}$, where they found Pseudomonas sp to accumulate maximum PHA at $\mathrm{pH}-7$, temperature $30^{\circ} \mathrm{C}$ in $2 \%$ carbon source.

Peak was obtained between 220 and 240 $\mathrm{nm}$ and confirmed the presence of PHA which indicated the presence of ester group. Even Nair et al., ${ }^{22}$ who confirmed the presence of PHA by obtaining peak between $230-240 \mathrm{~nm}$. The presence of PHA was confirmed by the retention factor 0.8 after running TLC.

The region at 1723 corresponds to the stretching of $\mathrm{C}=\mathrm{O}$ in Pseudomonas aeruginosa was found. Other absorption bands at 1363, 1458, 1723, 2926-2954 and $3881 \mathrm{~cm}^{-1}$ for $\mathrm{CH} 3,-\mathrm{CH}_{2}, \mathrm{C}=\mathrm{O}$, $-\mathrm{CH}$

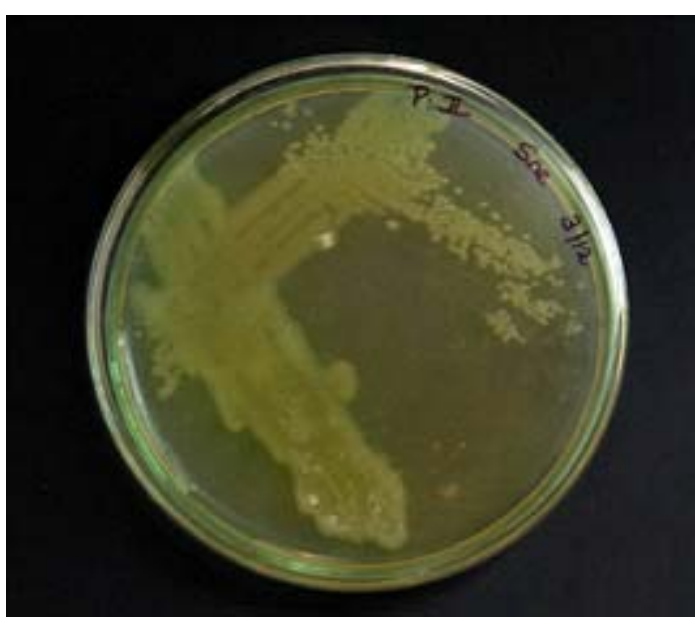

Fig.1. Growth of Pseudomonas aeruginosa on cetrimide agar

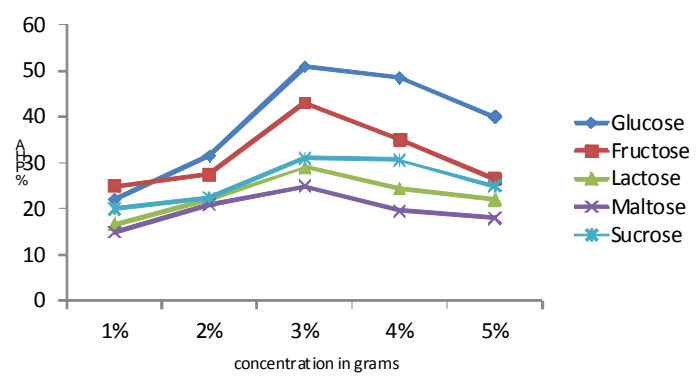

Fig.2. PHA accumulation in various carbon sources by Pseudomonas aeruginosa

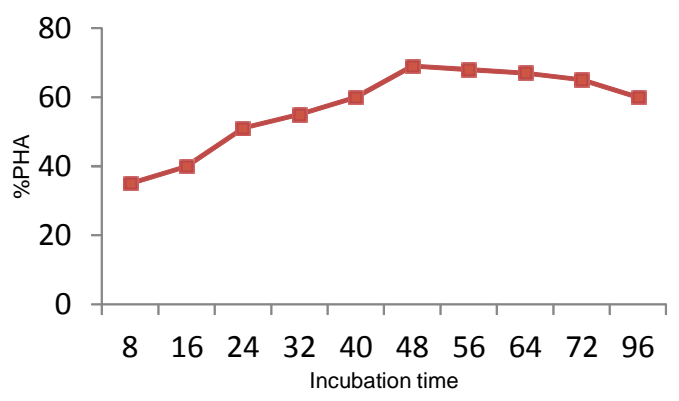

Fig. 3. PHA accumulation at various incubation time 


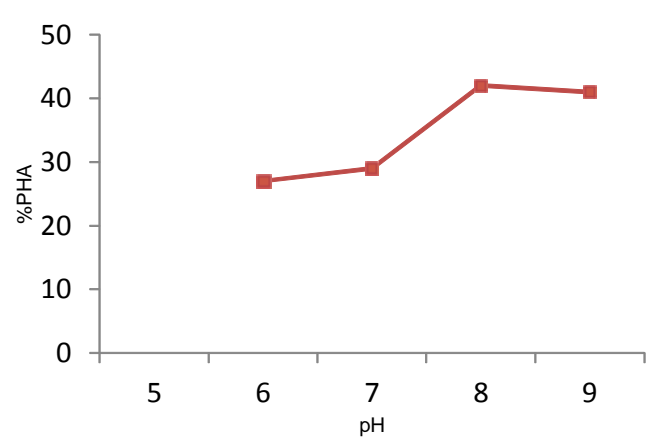

Fig. 4. PHA accumulation at different $\mathrm{pH}$

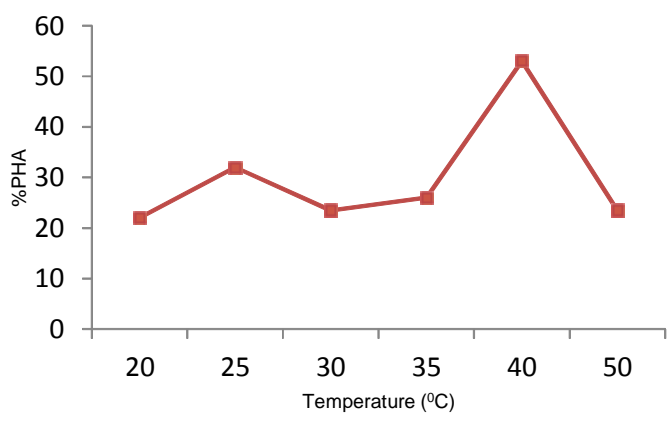

Fig.5. PHA accumulation at different temperature

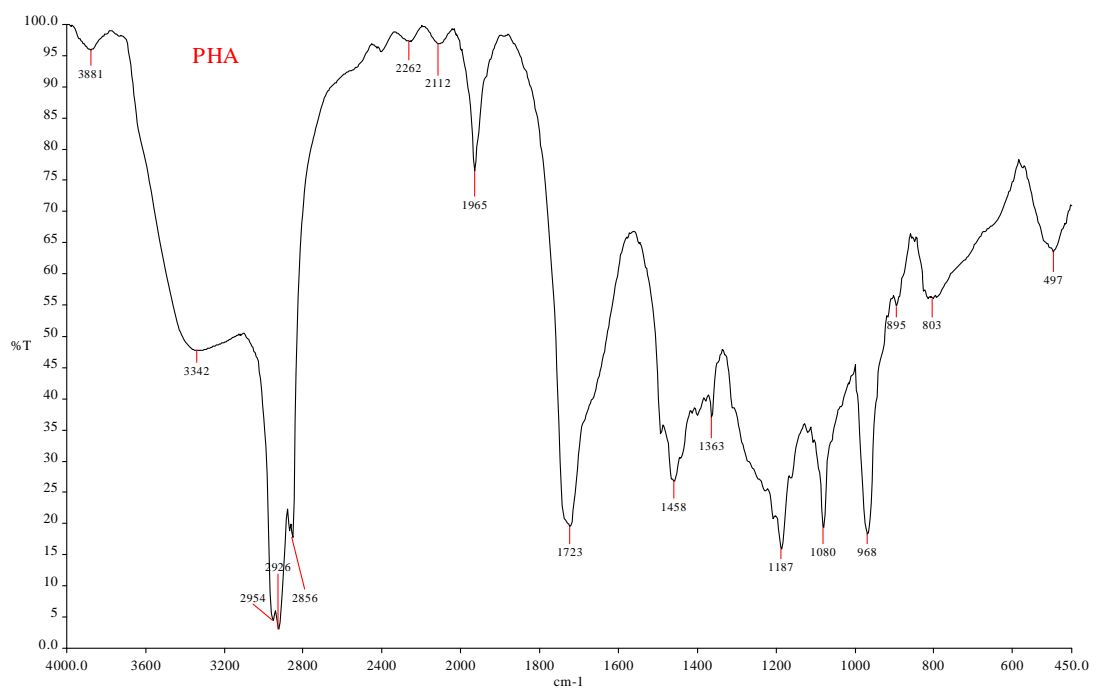

Fig. 6. FTIR analysis of PHA obtained from Pseudomonas aeruginosa

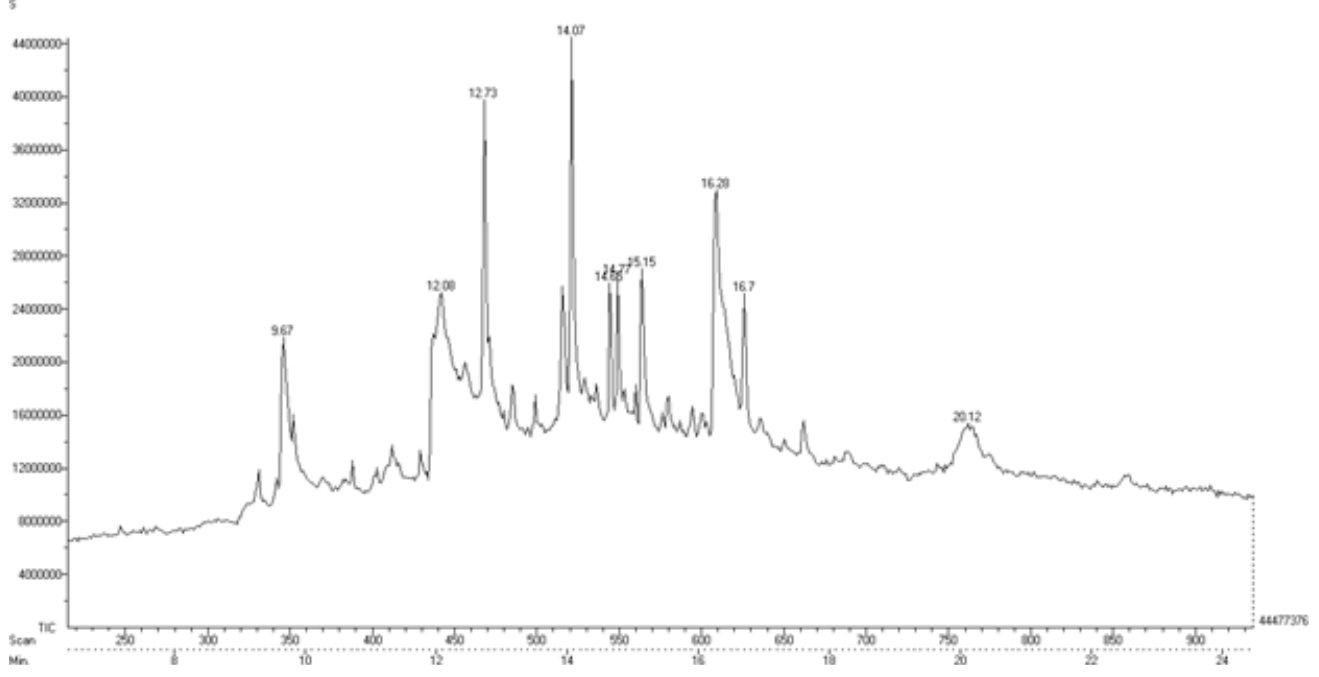

Fig.7. GCMS analysis of PHA obtained in sucrose containing medium from Pseudomonas aeruginosa 
and $\mathrm{OH}$ groups (Fig.6). This is on par with the result of Nisha et al., ${ }^{16}$ who found the peaks with the PHA isolated from Pseudomonas putida SU-8. The peaks at $14.07,15.15$ and 16.28 represents the presence of 3-hydroxytetradecatrienoate and 3hydroxyhexadecadienoate in Pseudomonas aeruginosa. (Fig 7). Mostly microbes accumulate either scl-PHAs with 3HB units or mcl-PHAs containing 3-hydroxyoctanoate (HO) and 3hydroxydecanoate (HD) as the major monomers ${ }^{2,9,10,17}$

\section{CONCLUSION}

Pseudomonas aeruginosa was found toaccumulate more PHA at $48 \mathrm{~h}$ incubation at $40^{\circ} \mathrm{C}$ in $3 \mathrm{~g} / 100 \mathrm{ml}$ in glucose containing medium at $\mathrm{pH}-8$ GC analysis of PHA of Pseudomonas aeruginosa to possess monomers of 3- hydroxytetradecatrienoate and 3-hydroxyhexadecadienoate.

\section{REFERENCES}

1. Macrae RM, Wilkinson JF. Poly-betahyroxybutyrate metabolism in washed suspensions of Bacillus cereus and Bacillus megaterium. J Gen Microbiol., 1958; 19(1): 210 2.

2. Anderson, A. J., \& Dawes, E. A. Occurrence, metabolism, metabolic role, and industrial uses of bacterial polyhydroxyalkanoates. Microbiological reviews., 1990; 54(4): 450-2.

3. Griebel, R. J., and J. M. Merrick. Metabolism of poly-hydroxybutyrate: effect of mild alkaline extraction on native poly-hydroxybutyrate granules. J Bacteriol., 1971; 108: 782-9.

4. D. Byrom, "Production of poly- $\beta-$ hydroxybutyrate: poly- $\beta$-hydroxyvalerate copolymers,” FEMS Microbiology Letters., 1992; 103: $247-0$.

5. Lillo, J.G., and Rodriguez-Valera, F. Effects of culture conditions on poly( $\beta$-hydroxybutyric acid) production by Haloferax mediterranei. Appl. Environ. Microbiol., 1990; 56: 2517-1.

6. Lemoigne $M$. Production of $\beta$-hydroxybutyric acid by certain bacteria of the B. subtilis group. Ann. Inst. Pasteur., 1925; 39: 144-6.

7. Braunegg G, Bona .R,and Koller. M Polymer Plastics Technology and Engineering, 2002; 43: 1-15.

8. Steinbuchel A. PHB and other polyhydroxyalkanoic acids: In G.R. H. J. Rehm, A. Puhler, and P. Stadler (ed.), Biotechnology: products of primary metabolism, 1st ed., vol. 6 . Wiley-VCH,Weinheim, Germany. 1995; 432434.

9. Lee SY. Biotechnol. Bioeng 1996; 49: 1-14.

10. Steinbuchel A. and Valentin H E, FEMS Microbiology Letter, 1995; 128: 219-228.

11. Law, J.H., Slepecky, R.A. Assay of poly- $\beta$ hydroxybutyric acid. J. Bacteriol., 1961; 82: 336

12. Jenifer S. A, Ahmed F, Jyotsana, P. Senthilkumar, and Antony V. Samrot. Accumulation Pattern of Polyhydroxyalkonoates by Agrobacterium tumefaciens SU-11 in Glucose Containing Medium. Journal of Pure and Applied Microbiology., 2014; 8 (6): 4817-1.

13. Samrot, A. V., Avinesh, R. B., Sukeetha, S. D., Senthilkumar, P. Accumulation of poly [(R)-3hydroxyalkanoates] in Enterobacter cloacae SU1 during growth with two different carbon sources in batch culture. Applied Biochemistry and Biotechnology., 2011; 163(1): 195-3.

14. de Rijk, T. C., van de Meer, P., Eggink, G., \& Weusthuis, R. A. Methods for Analysis of Poly (3 hydroxyalkanoate)(PHA) Composition. Biopolymers online. 2005.

15. Panda, B., Sharma, L., Singh, A. K., \& Mallick, $N$. Thin layer chromatographic detection of poly- $\beta$-hydroxybutyrate (PHB) and poly- $\beta$ hydroxyvalerate (PHV) in cyanobacteria. 2008.

16. Nisha, J., Mudaliar, N., Senthilkumar, P., \& Samrot, A. V. Influence of substrate concentration in accumulation pattern of poly (R) hydroxyalkonoate in Pseudomonas putida SU-8. African Journal of Microbiology Research., 2012; 6(15): 3623-0.

17. Brandl, H., Gross, R. A., Lenz, R. W., \& Fuller, R. C. Pseudomonas oleovorans as a source of poly ( $\beta$-hydroxyalkanoates) for potential applications as biodegradable polyesters. Applied and Environmental Microbiology., 1998; 54(8): 1977-1982.

18. Bhuwal, A. K., Singh, G., Aggarwal, N. K., Goyal, V., \& Yadav, A. Isolation and screening of polyhydroxyalkanoates producing bacteria from pulp, paper, and cardboard industry wastes. International Journal of Biomaterials, 2013.

19. Durner, R., Witholt, B., \& Egli, T. Accumulation of poly [(R)-3-hydroxyalkanoates] in Pseudomonas oleovorans during growth with octanoate in continuous culture at different dilution rates. Applied and environmental microbiology., 2000; 66(8): 3408-4.

20. Khanna, S., \& Srivastava, A. K. Recent advances in microbial polyhydroxyalkanoates. Process Biochemistry., 2005; 40(2): 607-9. 
21. Prasad M.P. and Sethi R. Production and isolation of polyhydroxyalkanoates from Pseudomonas sp using waste cooking oil as a sole carbon source. International Journal of Advanced Biotechnology and Research., 2013; 4(4): 527-2.
22. Nair, A. M., Annamalai, K., Kannan, S. K., Kuppusamy, S. Characterization of polyhydroxyalkanoates produced by Bacillus subtilis isolated from soil samples. Malaya $J$ Biosci., 2014; 1: 8-12. 